\title{
Féeries
}

Études sur le conte merveilleux, XVII $-\mathrm{XIX}{ }^{\mathrm{e}}$ siècle

$10 \mid 2013$

Conte et croyance

\section{Quand le conte nous fait croire en la science : le cas de Boureau-Deslandes}

When Tales Make Us Believe in Science: The Case of Boureau-Deslandes

\section{Guilhem Armand}

\section{(2) OpenEdition}

1 Journals

Édition électronique

URL : http://journals.openedition.org/feeries/897

DOI : $10.4000 /$ feeries.897

ISSN : 1957-7753

Éditeur

UGA Éditions/Université Grenoble Alpes

\section{Édition imprimée}

Date de publication : 20 septembre 2013

Pagination : 181-193

ISBN : 978-2-84310-253-0

ISSN : 1766-2842

\section{Référence électronique}

Guilhem Armand, "Quand le conte nous fait croire en la science : le cas de Boureau-Deslandes », Féeries [En ligne], 10 | 2013, mis en ligne le 20 mars 2015, consulté le 08 septembre 2020. URL : http:// journals.openedition.org/feeries/897 ; DOI : https://doi.org/10.4000/feeries.897

\section{(c) Féeries}




\section{QUAND LE CONTE NOUS FAIT CROIRE EN LA SCIENCE : LE CAS DE BOUREAU-DESLANDES}

$F$

ILS NATUREL " DES LUMIËRES, selon l'expression de J.-P. Sermain, le conte de fées «a pleinement intégré les analyses philosophiques et anthropologiques» de son époque. Et l'on sait combien le conte intègre les modèles et les méthodes des savoirs nouveaux ${ }^{\mathrm{I}}$, surtout durant ce que Jean-Paul Sermain a nommé la «troisième période» du conte de fées, celle des "diversifications", qui s'étend de 1730 à 1756 et qui se caractérise par un renouvellement du genre, de ses thèmes et de son écriture ${ }^{2}$ : le conte redéfinit notamment son lien aux superstitions, en rapport avec les combats des Lumières sur le progrès des connaissances.

Néanmoins, force est de constater que la science des Lumières demeure relativement rare dans ce genre. C'est que le conte de fées demeure pour beaucoup le genre de la légèreté - et la critique l'a longtemps ainsi conçu ${ }^{3}$, plus qu'il ne l'était en vérité —, que le merveilleux relève, par définition, d'une entorse aux lois physiques : en cela, il s'oppose radicalement au discours scientifique et à sa rigueur, tel qu'il se constitue tout au long de la période moderne ${ }^{4}$. D’une manière générale, si certains conteurs s'inspirent de l'actualité scientifique pour en quelque sorte alimenter un renouveau du merveilleux, à l'instar de Cahusac ou de Gautier de Montdorges,

I. Voir Féeries, n 6, 2009 : Le Conte, les savoirs.

2. J.-P. Sermain, Le Conte de fées du classicisme aux Lumières, Paris, Desjonquères, "L'esprit des lettres", 2005, p. 27-29.

3. Voir, par exemple P. Hazard qui résume le genre à une simple littérature d'évasion : «On ne reconnaît plus les lois du monde, que des pouvoirs magiques viennent bouleverser à plaisir; les corps ne sont plus pesants, les rêves sont vrais, la vertu est récompensée, le vice est puni. Quand on quitte enfin ces contes admirables, on trouve la vie si terne et si froide, qu'elle fait peine à vivre.» La Crise de conscience européenne, I680-I7I5, Paris, Fayard, "Le livre de poche», I96I, p. 338.

4. Sur ce point, nous nous permettons de renvoyer à notre ouvrage : Les Fictions à vocation scientifique, de Cyrano à Diderot, Bordeaux, PUB, "Mirabilia», 2013, notamment p. 33-64.

5. Sur ce point, voir l'article de F. Gevrey, «Fées cartésiennes ou fées newtoniennes», Féeries, $n^{\circ} 6,2009$. 
cette présence de la science dans le conte en reste bien souvent au stade de la "réfraction ${ }^{6} »$. Les exemples sont donc rares; le plus évident étant Micromégas de Voltaire, inspiré par la physique newtonienne. Moins connu ${ }^{7}$, Amilec, ce «roman-conte» de Tiphaigne de la Roche, constitue pratiquement un hapax dans le paysage tant littéraire que scientifique de l'époque, à l'image de son étonnant auteur : il s'agit d'une véritable fiction merveilleuse à vocation scientifique, c'est-à-dire qu'elle réussit l'alliance de la visée démonstrative du discours scientifique et de l'émerveillement propre au conte. C'est ce que permet le motif du rêve, que l'on peut lire comme un avatar de celui du voyage - généralement usité dans les fictions à vocation scientifique des $\mathrm{XVII}^{\mathrm{e}}$ et $\mathrm{XVIII}^{\mathrm{e}}$ siècles - en tant qu'il autorise le déplacement du discours sur un autre plan.

Dans L'Optique des mours opposée à l'optique des couleurs ${ }^{8}$, BoureauDeslandes fonde sa fiction orientale sur la vogue de l'optique newtonienne en $172^{9}$. Mais le déplacement géographique sert davantage la portée satirique du texte qu'une indécidable éventuelle visée démonstrative. En I74I, le même auteur avait fait paraître Pygmalion ou la statue animée, réécriture du mythe ovidien qu'il investissait, comme à son habitude, de sa verve satirique et libertine. Mais le libertinage de mœurs y rejoint clairement la philosophie matérialiste et sensualiste. Si la déesse Vénus est bien à l'origine de la métamorphose, elle semble juste fournir le moyen de représenter le passage de la matière inerte à la matière sensible, puis pensante, conformément à la thèse de l'auteur. Rappelons que Deslandes était un scientifique de formation, qu'il fut reçu élève géomètre à l'Académie des sciences en I7I2, membre de l'Académie des Sciences de Berlin, auteur de différents traités comme son Recueil de differens Traitez de Physique et d'Histoires Naturelles, propres à perfectionner ces deux sciences paru en I736, et surtout l'Histoire critique de la philosophie (I737). Contrairement à la statue de Condillac, par exemple, celle de Boureau-Deslandes se donne à lire comme un texte complet, et non comme un prologue imagé à un ouvrage de

6. Voir, A. Defrance «La réfraction des sciences dans le conte de fées», ibid.

7. Moins connu, certes, Tiphaigne est cependant de plus en plus étudié. Voir notamment l'article de P. Vincent dans Féries $n^{\circ}$ 6, les actes du colloque "Charles Tiphaigne (de la Roche) et les ambivalences du merveilleux moderne» organisé par Y. Citton à Grenoble les 25 et 26 mars 2010 (à paraître prochainement dans la collection «Mirabilia» des PUB), ainsi que le chapitre que je lui consacre dans "Les Fictions à vocation scientifique», p. 586-619. Signalons aussi qu'une équipe dirigée par Jacques Marx travaille actuellement à l'édition de ses œuvres complètes chez Garnier.

8. L'Optique des mours opposée à l'optique des couleurs, Londres, Samuel Harding, 1742. Signalons que le texte a été réédité dans la revue Féeries, nº 2, 2004-2005, p. 273-282, par B. Roukhomovsky.

9. U. Boskamp, «L'arc-en-ciel de Joseph-Marie Vien, Oracle d'une théorie de la couleur», dans T. W. Gaehtgens (éd.), L'Art et les normes sociales au XVIII' siècle, Paris, Éditions MSH, 200I, p. 32-33. 
sciences. Aussi pose-t-il davantage le problème du pacte de fiction entre le postulat de départ (ceci est un conte, donc faux, fabuleux, merveilleux...) et la volonté démonstratrice (qui se distingue du simple apologue, notamment quand le discours tend à une certaine vérité scientifique, au siècle des Lumières).

De plus, dans Pygmalion, la métamorphose magique et d'origine divine sert la thèse matérialiste de la sensibilité de la matière, autrement dit, c'est bien l'entorse aux lois physiques, constitutive du merveilleux, qui autorise l'insertion d'une démonstration scientifico-philosophique, voire qui permet au conte d'être, dans une certaine mesure, démonstration : l'entorse aux lois physiques n'en est pas une, la matière peut sentir et penser. Il s'agira donc ici d'analyser la double métamorphose de ce conte : la métamorphose qui est narrée, et celle que Deslandes fait subir à la fois au discours scientifique et à la valeur du merveilleux. On verra que l'on glisse d'un régime de croyance - celui du pacte merveilleux — à un autre - celui du discours scientifico-philosophique.

\section{Les métamorphoses du mythe}

L'épître dédicatoire liminaire présente le texte comme "cette histoire de Pygmalion que vous avez tant envie ou plutôt d'impatience de lire ${ }^{10} »$. Le mythe déjà bien connu se donne alors comme une histoire à découvrir et l'on glisse lentement vers le conte : peu importe que le sujet en soit fameux, comme chez Perrault, c'est le plaisir d'une narration qui entraîne la lectrice et le lecteur dans un univers où prime la séduction du merveilleux. Et la réécriture de la métamorphose ovidienne se caractérise d'abord par l'amplification. Les premières pages peignent un Pygmalion honnête homme. L'histoire se déroule dans une Antiquité de convention où Pygmalion vit heureux :

Dans l'Antiquité savante, l'apanage de ceux qui se livraient à l'étude des beaux-arts, était un noble désintéressement. Ils travaillaient pour la gloire, ils cherchaient à acquérir une réputation immortelle. La basse jalousie ne régnait point dans leurs cœurs. Pygmalion porta la générosité encore plus loin que tous ses contemporains" ${ }^{11}$.

Cette peinture pourrait presque évoquer l'âge d'or sans les pointes misogynes — lesquelles, on le sait depuis Perrault, relèvent d'une certaine forme

Io. A.-F. Boureau-Deslandes, Pygmalion ou la statue animée, dans Henri Coulet (éd.), Pygmalions des Lumières, Paris, Desjonquères, "XVIII ${ }^{\mathrm{e}}$ siècle», I998, p. 49. Abrégée en Pygmalion suivi du numéro de page, ce sera notre édition de référence pour cette étude.

II. Ibid., p. 5I-52. 
de galanterie - qui justifient, par la peinture des femmes du temps, le refus de l'amour par le héros, à peine esquissé en une phrase chez Ovide. On se situe bien alors davantage dans le cadre du conte que dans celui du mythe. L'on passe ainsi d'un type de fables - celles des Anciens, dirait Fontenelle - qui implique un certain type de croyance, à un autre : la fable moderne, aux contours plus flous, notamment chez Deslandes qui joue des différentes formes alors en vogue du conte. Lamplification de l'auteur permet un dialogisme entre passé et présent ${ }^{12}$ et place l'histoire dans ce hors-temps caractéristique du conte de fées. Et c'est tout naturellement Vénus qui joue en quelque sorte le rôle de la marraine bienfaisante permettant la naissance de l'amour :

Un jour de Printemps qu'il avait été seul à la campagne, où il s'était occupé à étudier la nature qui se réveillait, pour ainsi dire, et répandait partout de nouveaux agréments, il entra sans le savoir, dans un bosquet de myrtes consacré à Vénus. Là, saisi d'un mouvement inconnu, il se jeta sur un tapis de verdure émaillé de jonquilles et de violettes, et bientôt il s'y endormit. La déesse lui apparut en songe ${ }^{13}$.

Le topos du bosquet de myrtes et celui du songe évoquent le genre de la pastorale. Le sculpteur se trouve bien métamorphosé par cette vision d'une déesse lui commandant une statue : il ne s'agit pas seulement de lui obéir, mais de représenter «le beau dans sa source» tel qu'il le voit désormais («le véritable amour se peignit à son esprit»). Et le narrateur de commenter ce sentiment si parfait, rejoignant ainsi sur le fond et la forme la douce musique de l'églogue dans ces vers :

Tous les autres plaisirs ne valent pas tes peines!

Le vrai bonheur de l'âme est de porter tes chaînes.

Mais le texte renoue vite avec l'humour léger du conte galant; lorsque Pygmalion se met à l'œuvre et se sent même plus doué que d'ordinaire, Deslandes explique ses merveilleuses capacités au moyen d'une petite pointe misogyne :

Mais, pour cette fois, il sentit quelque chose de supérieur à son art. La déesse voulait réussir, et les femmes réussissent toujours, quand l'intérêt de leur beauté et l'amourpropre s'en mêlent ${ }^{14}$.

I2. «Le dialogisme du conte fait que le passé accueille la voix du présent, et le présent celle du passé», J.-P. Sermain, Métafictions (I670-I730), La réflexivité dans la littérature d'imagination, Paris, Champion, «Les Dix-huitièmes siècles», 2002, p. 37I.

13. Pygmalion, p. 54.

14. Ibid., p. 56. 
La statue achevée provoque l'admiration de tous et, bien sûr, la jalousie des femmes. L'admiration de son œuvre par le sculpteur entraîne le désir qui se concrétise dans l'union des corps de la statue animée et de son créateur. L'écriture fine et légère s'autorise alors une gradation dans la sensualité et l'érotisme, qui frise par endroits une certaine grivoiserie; par exemple, lorsque Pygmalion, après lui avoir fait faire l'apprentissage des plaisirs charnels, présente la statue à ses amis, celle-ci éblouit la compagnie, notamment par la candeur de son expression :

Je ne sais quel air de naïveté et d'innocence, si cependant l'innocence peut subsister avec les épreuves qu'elle avait essuyées, je ne sais, dis-je, quel air de naïveté se répandait sur toutes ses actions, et sur toutes ses paroles ${ }^{15}$.

Cette fusion des corps peut aussi avoir une dimension métatextuelle et faire écho à ce que J.-P. Sermain nomme «le fantasme de l'écrivain ${ }^{16}{ }$. Le héros peut encore plus être vu comme un double de l'écrivain dans les réécritures du mythe de Pygmalion qui «abondent au XVIII ${ }^{\mathrm{e}}$ siècle» parce que «ses artistes rêvent d'une perfection imitative dont ils seraient récompensés par une étreinte aimante, accordée par l'œuvre devenue vivante ${ }^{17} »$ comme l'explique J. Starobinski.

Si le XIX ${ }^{\mathrm{e}}$ siècle préfèrera le mythe de Prométhée, c'est-à-dire la création imitative conçue comme un défi puni par les dieux, la créature se rebellant contre son créateur — l'on pense alors à Frankenstein — la prééminence de la figure de Pygmalion au siècle des Lumières témoigne davantage d'un désir de récompense qui s'accorde, chez Deslandes particulièrement, avec un éloge libertin de la sensualité qui place le corps au cœur de toute réflexion. Cette conception du savoir, sensualiste à plus d'un titre, se manifeste dans la prise d'autonomie du personnage de la statue qui prenant conscience d'elle-même affirme sa liberté. À l'idée stéréotypée de la prison d'amour évoquée dans les premières pages, elle répond à la fin par le refus du mariage si attendu dans le conte de fées, au profit d'une conception libertaire de l'amour :

Pour nous jurer l'un à l'autre que nous vivrons toujours ensemble, sommes-nous assurés que nous nous plairons toujours? Pourquoi vouloir percer dans un avenir incertain? Je vous jure, moi, que tant que vous me plairez, je ne vous abandonnerai point : je vous jure de plus, que je ferai tous mes efforts pour vous plaire toujours.

15. Ibid., p. 68-69.

16. J.-P. Sermain, Métafictions, ouvr. cité, p. 371-377.

17. J. Starobinski, Le Remède dans le mal. Critique et légitimation de l'artifice à l'âge des Lumières, Paris, Gallimard, «NRF essais», I989, p. 249. 
À ce prix aimons-nous. Laissez les serments à ceux qui n'en connaissent pas la force, aux fous et aux imbéciles ${ }^{18}$.

Le texte de Deslandes joue ainsi avec les différentes attentes du conte, se situant à mi-chemin entre le conte de fées et le conte galant, voire libertin. Il signale que la fable narrée avec légèreté a une tout autre portée. Et l'éloge du plaisir qui parcourt le récit, que ce soit dans les interventions du narrateur ou dans les répliques des personnages, prend bien l'accent de la philosophie épicurienne : créer un être, c'est l'inviter au plaisir, manifestation de sa liberté d'exister. Et en ce sens, l'intervention initiale de Vénus peut faire écho à l'éloge de la déesse au tout début du De Natura. Son apparition finale souligne l'importance de plaire à ce qu'on aime, plaçant ainsi le plaisir au centre de tout. La divinité, plus qu'un personnage merveilleux — comme la fée - a donc surtout une valeur allégorique dans cette fable épicurienne où le merveilleux réside davantage dans le processus d'animation de la statue, sur lequel insiste le sous-titre. Quand Deslandes réécrit le mythe originel sous la forme du conte de fées, il ne s'agit plus du mythe des Anciens auquel on ne peut plus croire (on pense alors aux travaux de Fontenelle), mais d'une réactualisation dont les accents badins font écho au genre souvent plus réaliste du conte galant. À l'image de la métamorphose de la statue, Deslandes fait se mouvoir son texte à travers les genres de la fable, déplaçant ainsi le régime de croyance, suggérant — à l'instar de la fable allégorique un autre sens, mais aussi et surtout une autre forme d'adhésion au récit. Mais ce glissement générique rend plus mouvant le mode de croyance dans le conte, d'autant plus que le récit se fait démonstration.

\section{Fable philosophique et démonstration scientifique}

L'épicurisme du conte ne se limite pas à la réécriture libertine et sensuelle du mythe ovidien. Jean Macary explique, à propos de ce texte, que «le philosophe prend alors le masque de la fiction ${ }^{19}$ » et A. Gaillard souligne même que

paradoxalement, chez Deslandes, c'est dans ce texte de fiction que la thèse de la matérialité et de la mortalité de l'âme est la plus explicite, plus clairement encore que dans l'Histoire critique de la Philosophie ${ }^{20}$.

18. Pygmalion, p. 69.

19. J. Macary, Masque et lumières au XVIII" : André-François Deslandes, "citoyen et philosophe", 1689-I757, volume 78 des Archives internationales d'histoire des idées, La Haye, Springer, I975, p. I44.

20. A. Gaillard, Le Corps des statues, Le vivant et son simulacre à l'âge classique (de Descartes à Diderot), Paris, Champion, 2003, p. 88. 
L'épître pose déjà les questions philosophiques auxquelles répondra le conte: "Qu' est-ce que la matière? En quoi consiste son essence? Avouons-le de bonne foi : nous n'en savons rien ${ }^{21}$." L'aveu initial d'ignorance semble autoriser a priori la suspension du jugement propre au conte. Mais sous couvert de la fantaisie d'un récit merveilleux, Deslandes propose en fait une sorte d'expérimentation par l'imaginaire. Il s'agit de donner à voir la progression de la matière inerte vers la pensée, en passant par la matière animée, comme le fera plus tard Diderot dans Le Rêve de d'Alembert, ou Condillac dans le Traité des sensations. Mais ce qui n'est qu'un épisode chez l'un, une fable liminaire, prologue d'un ouvrage savant chez l'autre, se donne à lire chez Deslandes comme un texte complet. Le surnaturel prend implicitement le relais du discours scientifique qui ne peut répondre à ces questions par une véritable expérimentation. Si le pacte de fiction implique une adhésion principalement sur le mode de la croyance, la réflexion philosophique - qui prend place dans le conte merveilleux — implique, elle, une adhésion sur le mode de la réflexion. L'interrogation de Pygmalion, ayant achevé sa sculpture et regrettant que ce ne soit qu'une statue, ne prend guère les accents de la plainte élégiaque, mais plutôt le ton de la réflexion philosophique :

Ce marbre sera toujours un objet charmant à mes yeux; mais il y aura toujours un vide infini entre son existence et la mienne. Qui peut communiquer la pensée et le sentiment à du marbre? Mais qui me les a communiqués à moi-même? [...] Ici la matière est étendue, là elle pèse, plus loin elle se meut, plus loin encore elle pense. Ce ne sont peut-être là que différentes modifications qui concourent à former un tout parfait $^{22}$.

Les questions que se pose Pygmalion prennent aussi la valeur d'interrogations rhétoriques du conteur adressées au lecteur — en écho à celles de l'épître liminaire — et contrebalancent la suspension de jugement nécessaire au conte : il s'agit de réfléchir de concert sur la nature de la matière. Et la dernière hypothèse, formulée "nonchalamment" par le sculpteur, est justement le point de départ de la démonstration au cour de l'ouvrage qui, loin d'éveiller la statue comme par enchantement, reproduit toutes ces étapes au fur et à mesure, à commencer par le mouvement :

Comme le mouvement est le milieu par où doit passer la matière, pour, de nonpensante qu'elle était devenir pensante, la statue ne manqua point d'acquérir par degrés tous les mouvements dont un corps est susceptible ${ }^{23}$.

2I. Ibid.

22. Pygmalion, p. 58.

23. Ibid., p. 60. 
Mais le sculpteur attend impatiemment, depuis le début, le passage à la matière pensante :

Il épiait, pour ainsi dire, le moment favorable où sa statue cessait de l'être, où la matière étendue devait passer à un état plus parfait ou du moins plus perfectionné, où elle devait penser. Ce changement ne se fait point brusquement et par sauts : il se fait par degrés, par nuances, par des mouvements insensibles ${ }^{24}$.

Et la statue reproduit alors, en accéléré, l'évolution de l'être vivant, depuis la naissance jusqu'à l'être sentant, pensant et conscient, comme le rappelle la métaphore anticartésienne ${ }^{25}$ de l'"enfant au berceau»:

C'est ainsi qu'un enfant au berceau ressemble à quelque chose de brut, et de plus brut encore que du marbre. La machine se développe peu à peu, ses ressorts jouent les uns contre les autres, les fluides et les solides se combattent tour à tour, c'est une action et une réaction continuelle. [...] Ensuite la machine décroît, s'use, se détraque, périt. Lâme ressent les mêmes diminutions : elle n'était d'abord rien, elle devient quelque chose, elle se fortifie; elle retombe peu à peu dans un état d'anéantissement, elle s'anéantit enfin. Voilà la vie de l'âme, peu différente de la vie du corps. Il ne faut point qu'on s'y trompe ${ }^{26}$.

Dans ce conte qui débute véritablement avec l'apparition de Vénus et se clôt de même, l'auteur développe sa conception athée de l'existence ${ }^{27}$ : l'âme est mortelle, la matière est vivante et dans un cycle perpétuel où «tous [les] êtres n'en composent qu'un seul, qui est le Tout ${ }^{28} »$. Derrière le conte badin, le sujet est des plus sérieux et des plus polémiques en cette première moitié du XVIII ${ }^{\mathrm{e}}$ siècle. Une fois sa statue éveillée, naturellement douée de langage — manifestation de la pensée —, Pygmalion tente de lui expliquer ce qu' elle ignore, à savoir ce qu'est la nature, ce qu'est une divinité, mais il peine énormément dans ses explications à lui faire comprendre ce que signifie «vivre» :

24. Ibid., p. 59 .

25. Sur ce point, voir aussi l'article de S. Drouin, «Représentation de la perception et empirisme dans Pigmalion, ou la statue animée d'André-François Deslandes", dans Savoirs et fins de la représentation sous l'Ancien Régime, A. Cloutier, C. Dubeau et P.-M. Gendron (éd.), Cahiers du CIERL $\mathrm{n}^{\circ}$ I, Presses de l'Université de Laval, 2006, p. I3I-I4O; ainsi que A. Deneys-Tunney, «Le roman de la matière dans Pigmalion ou la statue animée (174I) d'A.-F. Boureau-Deslandes», dans B. Fink et G. Stenger (éd.), Être matérialiste à l'âge des Lumières : hommage offert à Roland Desné, Paris, PUF, «Écriture», 1999, p. 93-108.

26. Pygmalion, p. 59-60.

27. Pour un développement concernant la dimension scientifique de l'œuvre, nous nous permettons de renvoyer à notre chapitre sur Boureau-Deslandes : Les Fictions à vocation scientifique, ouvr. cité, p. 578 et suiv.

28. Pygmalion, p. 6 I. 
À proprement parler, répondit Pygmalion, tout vit, et ce qui paraît cesser de vivre, revit d'une autre manière. Mais pour vous expliquer des choses si sublimes, il faudrait entrer dans beaucoup de détails qui vous sont inconnus ${ }^{29}$.

C'est que la statue animée et pensante n'a pas encore atteint le stade de la conscience. C'est là que la réécriture libertine opère complètement, palliant en quelque sorte par un éloge de la volupté les oublis du mythe ovidien où la question ne se pose pas - du moins pas en ces termes. Observant sa "gorge avant-courière d'autres beautés plus secrètes», Pygmalion se laisse aller et l'embrasse. Le plaisir immédiat, sans l'entrave de la pudeur chez cette statue qui ne connaît pas le péché originel, fait naître la conscience de la Galatée moderne :

Que prétendez-vous, s'écria-t-elle, et quels mouvements inconnus me faites-vous sentir? Je me connais encore moins que je ne le faisais il y a quelques heures. À peine je vis et vous voulez que je meure ${ }^{30}$.

«Dans une conception toute sensualiste, c'est donc par l'apprentissage du plaisir et du plaisir amoureux que s'effectue l'acquisition des connaissances ${ }^{31}$ ", note Aurélia Gaillard, et, en effet, le doute aux consonances cartésiennes de la statue se lève grâce à l'acte d'union charnelle : «Il semblait que les secousses réitérées de cette espèce de plaisir augmentaient, pour ainsi parler, et perfectionnaient son âme ${ }^{32}$." Le conte galant sert l'expérimentation scientifique, le récit fictif est le medium de la démonstration, conférant alors à celle-ci la séduction du premier. Dès lors le sculpteur initie sa nouvelle compagne à la volupté, en commençant par une histoire du plaisir qui se moque ouvertement de la Genèse : «[...] on l'a masqué sous différents emblèmes. Le principal est celui d'une pomme, qui contenait la science du bien et du mal ${ }^{33}$." Car le plaisir est bien le principe même de la vie, comme le formule cartésiennement la statue au moment de la jouissance :

Je vis certainement, puisque j'en suis enivrée. Mais comment a-t-on pu connaître que le plaisir était caché dans ce réduit aimable, où vous me l'avez fait sentir? Comment a-t-on pu pénétrer cet aimable mystère ${ }^{34}$ ?

29. Ibid., p. 62.

3o. Ibid., p. 63-64.

3I. A. Gaillard, Le Corps des statues, ouvr. cité, p. 90.

32. Pygmalion, p. 64.

33. Ibid.

34. Ibid. 
L'expérimentation empiriste balaie la morale et ainsi redéfinit la quête du savant comme cet art de "pénétrer cet aimable mystère» et de renouveler l'expérience :

Mais pendant qu'ils s'entretenaient ainsi l'un de l'autre, le doux sommeil, qui est une récompense du plaisir généreusement porté à quelque excès, vint les surprendre. Pygmalion s'endormit dans les bras de son admirable statue, qui à son tour se refusa quelques instants au sommeil comme à une espèce d'anéantissement, et qui s'y livra ensuite comme à un état d'indolence qui renouvelle les forces et le goût du plaisir ${ }^{35}$.

Les voluptés de l'amour se conjuguent ainsi à la plus douce philosophie : sentir le désir c'est ressentir que l'on existe, éprouver le plaisir c'est savoir que l'on vit. Par cette fusion du discours érotique et du propos philosophicoscientifique, Boureau-Deslandes a révélé qu'une forme de transcendance se pouvait concevoir dans ce matérialisme athée où aimer et démontrer sont synonymes.

Le mythe, ainsi réécrit, corrige la fable religieuse. Ce faisant, il glisse du conte vers la fiction à vocation scientifique et philosophique, substituant un savoir dont il a fait la démonstration — du moins dont il a proposé la représentation - à un autre qui se trouve relégué au rang de croyance fausse. L'expérimentation par l'imaginaire repose sur le pouvoir magique de Vénus qui peut alors valoir pour une allégorie du pouvoir de la science : la merveille, dans ce texte, réside, principalement dans un processus d'accélération temporelle, puisque «le temps n'est rien pour la nature ${ }^{36}$ " comme le soulignera plus tard D'Alembert en voyant Diderot broyer la statue de Falconet. La fiction — dont vraisemblablement l'auteur du Rêve de D’Alembert se souviendra — permet juste une accélération temporelle qui permet de constater de visu les «degrés ", "nuances» et «mouvements insensibles» de cette évolution de la matière. La fable autorise un déplacement du point de vue et le sur-naturel consiste finalement en une vision surplombante de ce qui se produit plus lentement dans la nature. Le récit prend alors la portée d'une allégorie scientifique où le pacte merveilleux repose en fait sur la thèse matérialiste et inversement. La réécriture du mythe devient le truchement de l'exposition d'une conception de la matière, et même d'une observation expérimentale du passage de l'inerte au vivant, à l'instar du voyage interplanétaire qui se donne comme une démonstration de la cosmologie galiléenne ou newtonienne chez Cyrano ou Voltaire.

35. Pygmalion, p. 65.

36. D. Diderot, Le Rêve de D’Alembert, C. Duflo (éd.), Paris, Flammarion, «GF», 2002, p. 62. 


\section{Plaisir, pensée, croyance}

L'empirisme radical ${ }^{37}$ de Deslandes justifie le recours à la fiction. Celle-ci met en abyme sa propre épistémologie dès l'épître liminaire. L'auteur y formule une remise en question qui porte aussi bien sur la diégèse du conte que sur la théorie matérialiste à laquelle ce dernier se rattache, ou plutôt à laquelle le mythe réécrit au XVIII ${ }^{\mathrm{e}}$ siècle se trouve relié :

Ces traits sont excusables dans un sujet aussi bizarre et aussi philosophique (l'un n'est point contraire à l'autre) que celui que je vous présente. En effet, Madame, quel mélange d'objets inespérés et frappants! Un homme amoureux de son ouvrage : une statue vivante et animée : de la matière qui passe par plusieurs essais, qui reçoit différentes modifications, qui se meut, qui a des sentiments : une divinité puissante qui lui accorde jusqu'à la faculté de penser et de raisonner ${ }^{38}$ !

Sur le même plan, théorie et fiction sont soumises au doute raisonnable. Le pacte merveilleux peut-il valoir pour un protocole expérimental dans ce texte qui se donne aussi bien pour un conte que pour l'exploration d'un «sujet [...] philosophique»? La mise en abyme se poursuit dans le récit et à plusieurs niveaux. Le personnage principal souligne les limites de la raison :

Pygmalion rappelant toutes ses idées, se disait avec une sorte de confiance : Mais quoi! ma raison ne voit rien à tout cela. Ma raison! Qu'est-ce que ma raison, et que voit-elle? Que m’a-t-elle appris depuis que je suis au monde? Quelles ténèbres a-t-elle dissipées? Qu'est-ce qu'un homme raisonnable? Quel avantage a-t-il sur ceux qu’on suppose ne l'être point ${ }^{39}$ ?

L'expression du doute, qui surgit peu après la «commande» de Vénus, est assez paradoxale puisqu'elle s'opère "avec une sorte de confiance». Cette assurance naît donc du constat de l'inutilité de la raison et peut avoir la portée métatextuelle d'une justification du recours à la fiction et au merveilleux dans le cadre d'une observation expérimentale. Ce n'est plus à la raison de voir, mais à une autre faculté, l'imagination, ce n'est plus à la science moderne et rationnelle de démontrer, mais au conte. On glisse ainsi du régime rationnel à celui de l'imaginaire et ainsi, de l'adhésion raisonnée à la croyance. L'enthousiasme du sculpteur peut en effet s'apparenter à l'expression d'une foi - étonnante dans un texte matérialiste - mais qui porte finalement sur les potentialités de la matière :

37. Voir J. Macary, ouvr. cité, p. I6I.

38. Pygmalion, p. 49.

39. Ibid., p. 59. 
Qu'étais-je dans le premier instant où j'ai commencé à penser et à sentir? Que suis-je encore maintenant? Je vis, je respire, je pense, j'ai des sentiments : n'en peut-il point arriver autant à cette statue ${ }^{40}$ ?

Le doute, ensuite, est aussi celui de la statue accédant à la pensée :

Après tous ces préliminaires, se déclara la pensée, comme un trait de lumière qui éclate dans une nuit obscure. La statue, non plus statue, pensa, et dans le même moment elle s'écria : Que suis-je et qu'étais-je il n'y a qu'un instant? Je ne me comprends point : je ne me connais point ${ }^{4 \mathrm{I}}$.

Et la pensée fut, mouvement quasi naturel de la matière s'animant par degrés, laissant momentanément dans l'oubli le personnage de Vénus : la merveille est inhérente à la nature. Ce questionnement est d'autant plus intéressant qu'il détourne le Discours de la méthode : il s'agit de prêter à un être de fiction l'interrogation cartésienne. Et, plus loin, lorsque la statue accède à la conscience de soi, elle l'exprime selon une formulation qui rappelle, une fois de plus, Descartes : "À présent, disait-elle, je ne puis douter que je ne vive. Ce que vous appelez plaisir achève de me convaincre de mon être, et de me persuader de sa réalité ${ }^{42}$.» Il s'agit là d'un véritable cogito libertin et sensualiste. Le plaisir n'est pas seulement un principe de vie, mais il est ce qui ôte le doute, il devient le fondement du savoir.

Le discours scientifico-philosophique, à savoir la théorie matérialiste présente en arrière plan et s'illustrant par le conte, contribue à donner au texte fictionnel une force philosophique. Nul besoin d'intervention narratoriale : la voix du conteur présente au début du texte s'estompe pour laisser la statue parler d'elle-même, et ainsi montrer la matière qui se met à penser. La voix du conteur disparaît peu après l'apparition de Vénus, comme pour suggérer que les instances créatrices du conte merveilleux - au niveau narratif comme au niveau diégétique - ne sont plus nécessaires. D’où la dimension paradoxale de l'épître liminaire qui vaut autant pour un pacte de fiction que pour un protocole expérimental : tout en subtilité, la structure du conte de Boureau-Deslandes vaut pour le "pourquoi non?» de Cyrano de Bergerac ou de Fontenelle. Le pacte merveilleux sert ici la mise en place d'une expérience par l'imaginaire dont l'accréditation passe alors à la fois par l'adhésion textuelle (au conte, le temps de la narration) et par la croyance intellectuelle (plus durable, car fondée sur une réflexion partagée). Grâce à la mise en abyme du doute, la fiction intègre une véritable

40. Ibid., p. 58.

4I. Ibid., p. 60.

42. Ibid., p. 64 . 
réflexion épistémologique. Elle permet de penser, à l'intérieur du conte, les conditions d'adhésion ou de croyance à la fable. En doutant du pouvoir de la raison, en mettant en scène cette réfutation à travers le personnage de Pygmalion, puis de la statue, elle souligne l'importance de l'imagination. Il ne s'agit plus seulement de montrer un «mélange d'objets inespérés et frappants» susceptible d'étonner la lectrice, mais de démontrer par une accélération temporelle comment la matière inerte prend vie.

Le pacte de lecture est donc fondé sur un paradoxe : si le mythe est faux, la théorie demeure vraie, du moins le conte incite à sa croyance, même le récit merveilleux achevé, même close l'adhésion à la fiction du il était une fois. Enclose entre les seuils que constituent les apparitions de Vénus, la monstration du processus acquiert la force d'une révélation.

Pygmalion ou la statue animée n'est pas tant le récit d'une statue qui s'anime par la volonté d'une déesse, que la monstration du pouvoir de la matière d'acquérir vie, sensation et pensée. Ce paradoxe est redoublé par la narration qui part de l'intervention divine pour appuyer une thèse matérialiste : la science deviendrait alors un objet de croyance. L'on glisse ainsi, par le truchement du pacte merveilleux, d'un système de croyance périmé — le mythe - à un autre : la philosophie. Et la philosophie de Deslandes, telle qu'elle apparait dans ce texte fait la synthèse entre le fond — la thèse matérialiste - et sa méthode qui repose sur la soumission du rationnel à l'imaginaire. C'est une véritable épistémologie du plaisir comme marqueur de vérité qui se met en place ici.

Quel lieu plus propice que le conte, en effet, pour donner à concevoir comment de l'inerte peut émerger le plaisir? L'équation de l'imagination et du plaisir justifie le désir d'y croire du lecteur. Le principe du conte déborde du pacte fictionnel : la déesse s'estompe pour ne plus finalement laisser que la matière, celle du livre et du lecteur. Comme l'imagination de ce dernier a pu donner vie au récit contenu dans les feuilles, la pensée peut bien naître de l'inerte. 